\title{
QUEEN'S
UNIVERSITY
BELFAST
}

\section{Latent class analysis of ARDS subphenotypes a secondary analysis of the statins for acutely injured lungs from sepsis (SAILS) study}

for the NHLBI ARDS Network (2018). Latent class analysis of ARDS subphenotypes a secondary analysis of the statins for acutely injured lungs from sepsis (SAILS) study. Intensive Care Medicine, 44(11), 1859-1869. https://doi.org/10.1007/s00134-018-5378-3

Published in:

Intensive Care Medicine

Document Version:

Peer reviewed version

Queen's University Belfast - Research Portal:

Link to publication record in Queen's University Belfast Research Portal

Publisher rights

Copyright 2018 Springer. This work is made available online in accordance with the publisher's policies. Please refer to any applicable terms of use of the publisher.

\section{General rights}

Copyright for the publications made accessible via the Queen's University Belfast Research Portal is retained by the author(s) and / or other copyright owners and it is a condition of accessing these publications that users recognise and abide by the legal requirements associated with these rights.

Take down policy

The Research Portal is Queen's institutional repository that provides access to Queen's research output. Every effort has been made to ensure that content in the Research Portal does not infringe any person's rights, or applicable UK laws. If you discover content in the Research Portal that you believe breaches copyright or violates any law, please contact openaccess@qub.ac.uk. 


\title{
Latent Class Analysis of ARDS Subphenotypes: A Secondary Analysis of the Statins for Acutely Injured Lungs from Sepsis (SAILS) Study
}

\author{
Pratik Sinha ${ }^{1}$, Kevin L. Delucchi ${ }^{2}$, B. Taylor Thompson ${ }^{3}$, Daniel F. McAuley ${ }^{4,5}$, Michael A. \\ Matthay ${ }^{1,6,7}$, and Carolyn S. Calfee ${ }^{1,6,7}$ for the NHLBI ARDS network
}

\author{
Affiliations: \\ 1 Department of Medicine, Division of Pulmonary, Critical Care, Allergy and Sleep Medicine; University \\ of California, San Francisco; San Francisco, CA \\ 2 Department of Psychiatry; University of California, San Francisco; San Francisco, CA \\ 3 Department of Medicine, Division of Pulmonary and Critical Care, Massachusetts General \\ Hospital, Boston, Massachusetts, USA \\ 4 Centre for Experimental Medicine, School of Medicine, Dentistry and Biomedical Sciences, Queen's \\ University of Belfast, UK \\ 5 Regional Intensive Care Unit, Royal Victoria Hospital, Belfast, UK \\ 6 Department of Anesthesia; University of California, San Francisco; San Francisco, CA \\ 7 Cardiovascular Research Institute; University of California, San Francisco; San Francisco, CA \\ Corresponding Author: Dr Pratik Sinha \\ 505 Parnassus Ave, Box 0111 \\ San Francisco, CA 94143-0111 \\ Ph: 415-476-5756; email: pratik.sinha@ucsf.edu
}

Funding: HL131621, HL133390, HL140026 (CSC), 2T32GM008440-21 (PS); SAILS was supported by a grant from the National Heart, Lung, and Blood Institute, National Institutes of Health (HHSN268200 536165C-536179C), and the Investigator-Sponsored Study Program of AstraZeneca.

Word Count: 4128 (Abstract 254)

This article has an online data supplement. 


\begin{abstract}
Purpose: Using latent class analysis (LCA), we have consistently identified two distinct subphenotypes in four randomized controlled trial cohorts of ARDS. One subphenotype has hyper-inflammatory characteristics and is associated with worse clinical outcomes. Further, within 3 negative clinical trials, we observed differential treatment response by subphenotype to randomly assigned interventions. The main purpose of this study was to identify ARDS subphenotypes in a contemporary NHLBI Network trial of infection-associated ARDS (SAILS) using LCA and to test for differential treatment response to rosuvastatin therapy in the subphenotypes.
\end{abstract}

Methods: LCA models were constructed using a combination of biomarker and clinical data at baseline in the SAILS study ( $n=745)$. LCA modeling was then repeated using an expanded set of clinical class-defining variables. Subphenotypes were tested for differential treatment response to rosuvastatin.

Results: The 2-class LCA model best fit the population. $40 \%$ of the patients were classified as the "hyper-inflammatory" subphenotype. Including additional clinical variables in the LCA models did not identify new classes. Mortality at Day 60 and Day 90 was higher in the hyperinflammatory subphenotype. No differences in outcome were observed between hyperinflammatory patients randomized to rosuvastatin therapy versus placebo.

Conclusions: Using LCA, a 2-subphenotype model best described the SAILS population. The subphenotypes have features consistent with those previously reported in 4 other cohorts. Addition of new class-defining variables in the LCA model did not yield additional subphenotypes. No treatment effect was observed with rosuvastatin. These findings further validate the presence of two subphenotypes and demonstrates their utility for patient stratification in ARDS.

Key words: ARDS, Subphenotypes, Latent class analysis, Statins 


\section{Introduction}

Acute respiratory distress syndrome (ARDS) is defined as acute hypoxic respiratory failure $\left(\mathrm{PaO}_{2} / \mathrm{FiO}_{2}<300 \mathrm{mmHg}\right)$, bilateral chest infiltrates, and the absence of cardiac failure as the primary diagnosis. Despite 50 years of research, there are no successful disease-altering pharmacological interventions in the treatment of ARDS. Biological heterogeneity subsumed within this clinical syndrome is considered one of the main causes for failure of pharmacological interventions in randomized controlled trials (RCTs).[1]

Data from pre-clinical studies and non-RCTs have been highly encouraging for inflammationabrogating immunomodulatory therapies in ARDS. [2] Among these, 3-hydroxy-3-methylglutaryl coenzyme A reductase inhibitors (statins) had been shown to attenuate inflammation in murine lung injury models.[3] Pre-treatment with statins was also found to attenuate pulmonary inflammation in lipopolysaccharide-induced inflammation in healthy human volunteers, and prior statin therapy was associated with a survival benefit in patients with community-acquired pneumonia.[4, 5]. Two multi-center RCTs, hydroxymethylglutaryl-CoA reductase Inhibition with simvastatin in acute lung injury to reduce pulmonary dysfunction-2 study (HARP-2)[6] and rosuvastatin in sepsis-associated acute respiratory distress syndrome (SAILS) study [7], were recently conducted to test the hypothesis that statin treatment may be beneficial in treating ARDS. Both trials found no difference in clinical outcomes between patients treated with statin compared to placebo.

In prior studies, using latent class analysis (LCA) in four separate ARDS RCT populations, we have consistently identified two subgroups, which we have named the 'hypo-inflammatory subphenotype' and 'hyper-inflammatory subphenotype'.[8-10] The subgroups are clinically and biologically distinct from one another and remain stable over the first three days of enrollment.[11] The hyper-inflammatory subphenotype was associated with increased inflammatory biomarkers, a higher prevalence of shock, and worse clinical outcomes. Crucially, in the hyper-inflammatory subphenotype, we observed treatment responses to positive end- 
expiratory pressure (PEEP) and conservative fluid management that were significantly different from the hypo-inflammatory class.[9, 12] Most recently, in the HARP-2 study, we observed a survival benefit in the hyper-inflammatory subphenotype with simvastatin therapy.[13]

In the presented study, we hypothesized that in SAILS, a more contemporaneous and sepsisspecific cohort of ARDS, two subphenotypes would once again be identified. In addition, we wanted to test whether using additional clinical data as class-defining variables in the LCA models would lead to identification of new latent classes. Further, in line with the findings of the HARP-2 LCA, we hypothesized that rosuvastatin therapy would preferentially confer a survival benefit in patients with a hyper-inflammatory subphenotype.

Part of these results have been previously reported in the form of an abstract.[14]

\section{Methods}

\section{Study Design and Population}

This study was a secondary analysis of the National Heart, Lung, and Blood Institute (NHLBI) ARDS network randomized controlled (SAILS) trial. The original trial ran from March 18, 2010 to September 30, 2013 ( $n=745$ ). Aside from standard ARDS diagnostic criteria, patients were also required to have a known or suspected infection and one of the following: a white-cell count $>12,000 / \mathrm{mm}^{3}$ or $<4000 / \mathrm{mm}^{3}$ or a differential count with $>10 \%$ band forms, or core temperature $>38^{\circ} \mathrm{C}$ or $\left\langle 36^{\circ} \mathrm{C}\right.$. Patients were randomized to receive rosuvastatin $(40 \mathrm{mg}$ loading followed by $20 \mathrm{mg}$ daily) or placebo. Primary outcome for the trial was death before hospital discharge or 60-day mortality. There were no differences in outcome between the treatment and placebo groups in the original study.

Findings of this study were compared to our previous LCA in the HARP-2 trial.[13] HARP-2 was a multicenter randomized controlled trial conducted in the United Kingdom and Ireland comparing daily simvastatin $80 \mathrm{mg}$ to placebo in 540 patients with ARDS.[6] Unlike SAILS, recruitment in HARP-2 was not limited to patients with sepsis. Both trials were conducted over 
a similar time period. Full protocol details of both trials can be found in the original studies.[6, 7]

\section{Statistical Analysis}

\section{Latent Class Analysis (LCA)}

For the purposes of this study, two separate latent class analyses were performed. First, as with our previous studies[9, 12], we used a combination of clinical and biomarker data at baseline (prior to randomization)(Table S1). Four models were built consisting of 1, 2, 3, and 4 classes respectively. Optimal number of classes was evaluated using a combination of Bayesian information criteria, Vuong-Lo-Mendell-Rubin likelihood-ratio test, entropy, and number of observations in each class. Detailed explanation of LCA and the procedure for optimal model selection can be found in the supplementary material. Once identified, each individual was assigned a class according to model generated probabilities.

In comparison to previous NHLBI trials, the SAILS trial collected a more comprehensive list of variables. For the second latent class analysis, in addition to the variables used in the first LCA, these new variables were used as class-defining variables. The variables included presence of malignancy, recent immunosuppression ( $<6$ months), radiological lung injury score, smoking history, C-reactive protein, pre-morbid residence, and site of identified infection (Table S1). Additionally, chronic diseases with a sample prevalence of at least $10 \%$ were also included in the second LCA. Less prevalent diseases were considered unlikely to contribute to class definition and excluded from these models.

\section{Predicting class assignment using 3-variable model}

In our previous work, a biomarker-based 3-variable model was shown to have high accuracy in predicting subphenotypes.[9, 12] The availability in the SAILS dataset of C-reactive protein (CRP), a widely-used marker of inflammation and mechanistically downstream of IL-6, was leveraged to test whether subphenotypes could be accurately classified using CRP-based logistic regression models. In line with our prior work, a 3-variable model using CRP and two 
routinely measured clinical or laboratory variables that contributed most to defining subphenotypes was constructed. Next, the biomarker that contributed most to defining class was used alongside the two best clinical variables to construct a biomarker based 3-variable model. Receiver operator characteristics (ROC) and area under the curve (AUC) were calculated for the models and DeLong's test was used to compare model performance. Finally, to test the external validity of the identified subphenotypes across populations, we used the 3-variable (soluble tumor necrosis factor receptor-1, Interleukin-8, and serum bicarbonate) parsimonious logistic regression model developed in our prior study to assign class and compared the accuracy with LCA-derived subphenotype assignment.[9] Details of the procedure used for feature selection and logistic regression models can be found in the supplementary material.

Depending on distribution, data are presented either as mean ( \pm standard deviation) or median (interquartile range). Differences between groups were tested using Student t-test or MannWhitney $\mathrm{U}$ test. Differences in proportions were tested using chi-squared test. We used logistic regression analysis with mortality at 60 days and 90 days as the outcome. Ventilator-free days (VFD) was defined as the number of days the patient was alive and without ventilatory assistance to day 28. Death before day 28 was assigned no VFDs. Poisson regression was used to analyze the differential effect of rosuvastatin treatment versus placebo on VFDs across subphenotypes. Kaplan-Meier statistics were used to estimate 60-day survival with the population first stratified by subphenotype followed by treatment assignment. For this study, latent class models were fit using Mplus.[15] All other analyses were performed using RStudio version 1.0.143 ( http://www.rstudio.com/).

\section{Results}

\section{Identification of Optimal Latent Class Model}

Table 1 describes the fit statistics of the four LCA models generated. As anticipated, the Bayesian Information Criteria (BIC) decreased sequentially with addition of a new class to the models. The decrease in BIC was greatest, however, when moving from a 1-class to a 2-class model compared to models with more classes. Fit for the 2-class model was a statistically 
significant improvement compared to the 1 -class model $(p<0.0001)$. Three and four class models did not provide a significant improvement in model fit. Entropy was highest in the 2class model (0.84), suggesting strong class separation (Table 1). Based on these findings, the 2class model was judged to be the best fit for the population.

$448(60 \%)$ of the patients were assigned to class 1 , and 277 patients were assigned to class 2 (40\%). The median class assignment probabilities was 1.0 (IQR $0.98-1$ ) for Class 1 and 1.0 (IQR $0.96-1.0)$ for Class 2. A class probability of greater than 0.9 was seen in 403 (86.1\%) Class 1 patients and 232 (83.8\%) Class 2 patients, indicating strong class-differentiation of the model (Figures S1A and S1B).

\section{Characteristics and Outcomes in ARDS Subphenotypes}

As in our previous studies, Class 2 had characteristics in keeping with 'hyper-inflammation'; this class will be referred to as the 'hyper-inflammatory subphenotype' for the remainder of the manuscript. A greater proportion of the hyper-inflammatory patients had a primary diagnosis of non-pulmonary sepsis $(26.8 \%$ vs $15.2 \%, p<0.0001)$ and were on vasopressors at baseline $(81.6 \%$ vs $38.7 \%, p<0.0001$ )(Figure S2). On average, patients in the hyper-inflammatory subphenotype had lower systolic blood pressure $(77 \mathrm{mmHg}$ vs $87 \mathrm{mmHg}, p<0.0001)$, suggesting a greater proportion of these patients were in shock.

In comparison to the hypo-inflammatory subphenotype (Class 1), the hyper-inflammatory subphenotype had significantly higher mean values for plasma IL-6, IL-8, sTNF-R1, ICAM-1, and PAI-1. Levels for protein C were significantly lower in the hyper-inflammatory subphenotype. LCA modelling seeks to identify subgroups that are heterogeneous to each other, therefore, most variables were significantly different between the two subphenotypes (Table 2). Statistically, most standard laboratory variables were also significantly different between subphenotypes, however, only differences in bilirubin and creatinine were clinically significant. Higher values were observed in the hyper-inflammatory subphenotype for both these variables. In keeping with our previous studies, respiratory variables were mostly similar between the two 
subphenotypes. The relative contributions of the continuous variables in defining the subphenotypes are presented in Figure 1. The 3-variable biomarker-based regression model from our prior work demonstrated high accuracy in identifying LCA-derived subphenotypes in this cohort (AUC 0.95; Table S2), suggesting phenotype characteristics were similar to those identified previously.

Table 3A summarizes the differences in outcome between the two phenotypes. The hyperinflammatory subphenotype had a significantly higher 60-day mortality (36.5\% vs $20.9 \%, p<$ $0.0001)$, higher 90 -day mortality $(37.6 \%$ vs $21.4 \%, p<0.0001)$, and fewer ventilator-free days (15 days vs 23 days, $\mathrm{p}<0.0001$ ).

\section{LCA Models with Extended Class-Defining Variables}

When an extended set of class-defining variables were used in the LCA models, the 2-class model again best fit the population. In the extended 2-class model, there were 462 patients in the hypo-inflammatory subphenotype (6 fewer than initial model) and 283 patients in the hyper-inflammatory subphenotype (6 more than the initial model). Overall, in $98 \%$ of the patients the initial assignment of Subphenotype remained unchanged with the addition of more clinical data (Table 4).

In the extended variable LCA, thorax (pneumonia or aspiration pneumonitis) as the site of infection was seen more frequently in the hypo-inflammatory subphenotype $(85.8 \%$ vs $60.0 \%$, $p<0.0001$; Table 5), and the abdomen was the site of infection seen more frequently in the hyper-inflammatory subphenotype ( $15.2 \%$ vs $6.7 \%, p<0.0001$; Table 5$)$. Diabetes mellitus was also significantly more prevalent in the hyper-inflammatory subphenotype $(27.2 \%$ vs $20.3 \%$, $p=0.0310$ ), although the clinical significance of this difference remains uncertain (Table 5). Creactive protein (CRP) was significantly higher in the hyper-inflammatory compared to the hypo-inflammatory subphenotype ( $23.6 \mathrm{mg} / \mathrm{dL}$ vs $20.5 \mathrm{mg} / \mathrm{dL}, \mathrm{p}<0.001)$.

\section{Treatment Effect in Subphenotypes (Extended Variables LCA)}


There was no evidence for a subphenotype-specific treatment benefit for rosuvastatin. Specifically, there were no significant differences observed between the 60-day survival curves when the population were stratified by subphenotype and treatment (hypo-inflammatory $p=0.45$, hyper-inflammatory $p=0.33$; Figure 2 ). In the hyper-inflammatory subphenotype, no significant differences in outcome were observed between Rosuvastatin-treated and placebo groups in 60-day mortality, 90-day mortality, or ventilator free days to day 28 . There were no significant treatment effects observed in the hypo-inflammatory subphenotype (Table 3B). We also sought interactions between treatment response and subphenotypes. The analysis identified no significant interaction between subphenotypes and treatment allocation for either 90-day mortality $(p=0.95)$ or ventilator-free days $(p=0.70)$.

\section{Differences between HARP-2 (Simvastatin) and SAILS (Rosuvastatin)}

In contrast to HARP-2, we did not observe a subphenotype-specific treatment response in SAILS; therefore, post-hoc, we sought to compare the patient populations studied in the two trials. The mean $\mathrm{PaO}_{2} / \mathrm{FiO}_{2}$ at enrollment was significantly higher in SAILS compared to HARP-2 (167 vs $127 \mathrm{mmHg} ; \mathrm{p}<0.0001$ ). Despite being a trial that recruited only infection-related ARDS, the proportion of patients that were vasopressor-dependent was also significantly lower in the SAILS trial ( $54 \%$ vs $66 \%, p<0.0001)$, although high in both cohorts. Pneumonia was the primary risk factor for a higher percentage of patients in SAILS compared to HARP-2 ( $71 \%$ vs 58\%, $p<0.0001)$

\section{CRP-based regression model showed moderate performance in predicting subphenotypes}

Bicarbonate and creatinine were the two routinely measured clinical variables that contributed most to defining subphenotypes. These two variables in conjunction with CRP were used to construct a logistic regression model to identify class. Univariate analysis showed all three variables were predictors of subphenotype (CRP $p<0.001$, bicarbonate $p<0.0001$, creatinine $\mathrm{p}<0.0001$ ). The 3-variable regression model had a ROC AUC of 0.85 . Using the Youden index as the cut-off probability (0.41), the sensitivity of the model was 0.76 and specificity was 0.82 (Tables S3A and S3B). Soluble tumor necrosis factor receptor-1 (sTNFR1) was the biomarker 
that contributed most to class-definition. In comparison to the CRP-based model, the ROC AUC for a model consisting of sTNFR1, bicarbonate, and creatinine was significantly higher (AUC 0.93, $p<0.0001)$.

\section{Discussion}

This secondary analysis of the SAILS trial reports that using LCA, two distinct subphenotypes of ARDS best describe the population. As in our prior work, Subphenotype 2 has hyperinflammatory characteristics with increased mortality and fewer ventilator-free days. Findings from this study expand our knowledge of ARDS subphenotypes by confirming the relevance of the hyper-inflammatory subphenotype in a more specific (infection-related) and contemporaneous ARDS cohort. In addition, these data demonstrate that expanding the number of clinical variables in the LCA models did not alter the number of classes in the population, further illustrating the stability of the 2 observed ARDS subphenotypes. Unlike the LCA in the HARP-2 trial,[13] no treatment effect with rosuvastatin was observed in either subphenotype in this study.

LCA modeling in the SAILS cohort revealed findings similar to our previous studies. In total, using the same procedure, we have now reported the 2-class model optimally describing ARDS populations across four different NHLBI ARDS network datasets, namely, ALVEOLI, ARMA, FACCT, and SAILS. $[9,12]$ Of these, SAILS is the most recent study. The presented findings indicate that the two subphenotypes identified using LCA have contemporaneous relevance, despite changing demographics and practices in ARDS.[16] Moreover, the characteristics of the two subphenotypes have remained strikingly consistent across all datasets. Inclusion of several new variables into the model failed to identify further classes. Again, these findings strongly reinforce the validity of two subphenotypes in ARDS, as increasing the complexity of the model by incorporating additional class-defining co-variates is known to increase LCA performance.[17] The high predictive accuracy of a previously derived regression-based classifier model in SAILS further substantiates the validity of these phenotypes in randomized controlled trial cohorts of ARDS. Furthermore, replication of these findings in a fifth ARDS 
cohort from the UK and Ireland (HARP-2) albeit using a limited set of variables substantiate the robustness and generalizability of the two subphenotype model.[13]

A distinctive feature of the current study consistent with our previously presented work in ARDS subphenotypes $[9,12,13]$ is that respiratory variables, including the $\mathrm{PaO}_{2} / \mathrm{FiO}_{2}$ ratio $(\mathrm{PF}$ ratio) perform poorly at differentiating class (Figure 1). A recent study suggests stratification of patients by severity of ARDS using a cut-off of $\mathrm{PaO}_{2} / \mathrm{FiO}_{2}$ ratio of 150 may lead to identification of more homogeneous patients in ARDS with differing anatomy and physiology and would serve as a better threshold than the Berlin classification.[18] Interestingly, in SAILS, the incidence of patients with $\mathrm{PaO}_{2} / \mathrm{FiO}_{2}<150$ was similar in both subphenotypes $\left(\mathrm{PaO}_{2} / \mathrm{FiO}_{2}<150 \mathrm{mmHg}\right.$ : hypoinflammatory $60 \%$ vs hyper-inflammatory $64 \%, p=0.35)$. These findings further support the hypothesis that the presented LCA-based stratification of ARDS cannot be identified using failure of oxygenation alone. In other words, despite being subgroups of ARDS, the classes are not representative of severity of respiratory failure. These findings, however, must be interpreted with caution, given that the respiratory variables used in LCA did not include measurements of pulmonary dead space nor a more quantitative measure of pulmonary edema on the chest radiograph, both of which have been shown to predict outcome in ARDS.[19, 20]

Nevertheless, based on the presented study, the prominence of biomarkers such as IL-6, IL-8, sTNFR1, and Protein C as class-defining variables (Figure 1) suggests that the differences in identified classes may be primarily driven by underlying biology. The hyper-inflammatory subphenotype is associated with exaggerated inflammation, and as would be anticipated, the subphenotype is associated with hyperbilirubinemia, higher creatinine, and lower platelets (Table 2). Two factors suggest that the subphenotypes capture information beyond mere severity of organ-failure. First, organ failure scores, such as SOFA, are known to correlate poorly with pro-inflammatory cytokines in patients with sepsis.[21] Second, although SOFA and APACHE scores are useful in prognostication, the differential treatment responses observed in subphenotypes in our previous work were not identified when measures of severity were used 
to stratify the same population.[10] These findings suggest that the LCA approach to identifying subgroups cannot be reproduced using conventional measures of disease severity.

Although the biomarker and clinical data suggest a strong biological association with inflammation and the identified classes, evidence for a direct causal pathway is lacking. Currently, in line with best practice,[22] we have termed the classes "subphenotypes" because they represent subgroups within ARDS, itself a phenotypic entity. However, as the field develops, with further details emerging of the biological properties underlying the subgroups, it is likely that the nomenclature will change. In addition, should these subphenotypes be identifiable in critical illness syndromes beyond ARDS, then the nomenclature of the classes would also require revision to reflect their status as critical illness phenotypes. To that end, the lack of importance of respiratory variables in the model coupled with SAILS being a sepsisassociated cohort of ARDS suggests that these subgroups may not be exclusive to ARDS; it is possible that these subgroups are also applicable to sepsis, where inflammation is central to the underlying pathophysiology.

Several groups have used peripheral leukocyte gene expression to identify subgroups in sepsis patients admitted to the ICU.[23-26] Similar to the presented LCA subgroups, the subgroup identification in these studies is reliant on biological data. Direct comparison of subgroups we have identified using LCA with those identified in sepsis by transcriptomic analysis is not possible in the SAILS cohort due to the lack of corresponding transcriptomic data. Nonetheless, transcriptomic signatures of subphenotypes in ARDS and LCA in sepsis are worthy of future study.

Why might we have observed a subphenotype-specific treatment response to simvastatin in a previous study (HARP-2) but no similar treatment response to rosuvastatin in this analysis? There are several potential explanations. First, SAILS was specific for patients with infectionrelated ARDS, while HARP-2 enrolled patients with a wider variety of ARDS risk factors. This increases the possibility that sub-classes identified in SAILS differ from those identified in the 
HARP-2 study. This explanation seems unlikely, however, considering the remarkable similarity in the clinical and biomarker profile between our current and previously identified 'hyperinflammatory' class. Second, and perhaps the most likely explanation, may be differences in the type of statins used in the two trials. Despite the absence of pre-clinical data, the investigators in the SAILS trial used rosuvastatin as the statin of choice. This decision was based on the lower drug-drug interaction profile of rosuvastatin and an assumed class-effect across statins in ARDS. Simvastatin, however, is the only statin with clinical data supporting a role in ARDS, although atorvastatin also has some pre-clinical data in ARDS.[3, 5, 27, 28] Both are lipophilic molecules, whereas rosuvastatin is a hydrophilic molecule. Plasma protein expression of inflammatory biomarkers (IL-6, CRP), cortisol, and von Willebrand factor in response to statin therapy are known vary depending on whether the statin administered is hydrophilic or lipophilic.[29-31] Although rosuvastatin is known to have greater lipid-lowering potency than simvastatin,[32] this may not confer greater anti-inflammatory activity. In a population-based study of sepsis, Lee and colleagues have recently shown reduced mortality at 30 days with prior use of simvastatin and atorvastatin but not rosuvastatin.[33] Extrapolating these data to SAILS, where the benefits of rosuvastin were tested in sepsis-associated ARDS, the assumption of class-effect with all statins may not be valid. The choice of a hydrophilic statin in SAILS may have contributed to the negative result in this study compared to the secondary analysis of HARP-2.

A third possible explanation may be that the severity of ARDS, as defined by the $\mathrm{PaO}_{2} / \mathrm{FiO}_{2}$, was also different between the two trials. In a prospective observational study, Mansur and colleagues found that the protection afforded by statin therapy in ARDS was only observed in those patients with severe ARDS.[34] In addition to lower ARDS severity, the SAILS trial also had a relatively low mortality rate amongst patients with severe ARDS at baseline (23.7\%). Surprisingly, in the SAILS trial, 60-day mortality was highest in the mild ARDS population $(44 / 145,30.3 \%)$. If, as suggested, statin therapy is most effective in severe ARDS, the lower ARDS severity combined with lower mortality in the severe ARDS population may have contributed to the ineffectiveness of statin therapy in the SAILS trial and, consequently, within the hyper-inflammatory subphenotype. 
SAILS provided an opportunity to assess the value of C-reactive protein (CRP) for subphenotype identification. CRP is a widely used acute-phase reactant that is associated with increased mortality in critically ill patients.[35] CRP is known to be under regulatory control of numerous pro-inflammatory cytokines, with IL-6 being one of its chief regulators.[36] Given the importance of IL-6 as a class-defining variable (Figure 1), we anticipated CRP might be a useful variable to identify class. Despite the circular nature of the analysis, where the same data were used for model and dependent variable generation, the CRP-based models performed only modestly. In particular, compared to parsimonious models that incorporated biomarkers, the CRP-based model had inferior accuracy. It may be that, consistent with previous studies, absolute values of CRP are not reliable as indicators of severity of inflammation in ARDS or in critical illness.[37, 38] These findings suggest that research biomarkers such as sTNFR1, IL-6, or IL-8 are likely to be essential important components of accurate parsimonious classifier models that will need to be tested prospectively. The perturbing influence of anthropometric factors such as age and BMI on CRP that are not observed with IL-6 and IL-8, may in part explain this observation.[39] In addition, the higher incidence of liver impairment in the hyperinflammatory subphenotype may also lead to lower CRP levels compromising its utility as a classifier.[40]

This study has several strengths. LCA was performed using a data-driven unbiased approach. Enhanced complexity of class-defining variables in the LCA models adds to the robustness of the findings of two ARDS subphenotypes. The studied population of 745 patients was the most contemporaneous and comprehensive database in which we have applied LCA and represents a large cohort of infection-related ARDS patients. These results along with our previous work underline the generalizability of subphenotypes across varying ARDS populations.

This study also has limitations. The presented study is a secondary post-hoc analysis of an RCT, limiting any observations to inferential or associative. Prospective studies are needed before we can apply ARDS subphenotypes in clinical practice or clinical trials. The complexity of LCA 
models renders them impractical and inaccessible as clinical tools. Parsimonious models that identify subphenotypes using a limited set of variables are required to improve their clinical feasibility. Another rate-limiting step in identifying subphenotypes at the bedside is the inability to measure biomarkers rapidly, in real-time, and in a cost-effective manner. Technology that supports such measurements may be essential for subphenotype identification in the clinical setting. Constructing models that only utilize readily available clinical data may be useful and accelerate identification of subphenotypes in clinical practice and require further investigation. Finally, LCA subphenotypes have so far been limited to RCTs. A recent study, using alternative methods to identify subgroup in ARDS, suggests that a hyper-inflammatory subphenotype also exists in observational cohorts. [41] Further studies needed to confirm these findings using LCA.

\section{Summary}

This analysis confirms the existence of two discrete subphenotypes in a contemporary and specific subset of ARDS population. No treatment effect was observed with rosuvastatin therapy within subphenotypes. We postulate that this observation was due to RCT-design factors, and specifically, the choice of statin, rather than features of the subphenotypes, per se. The consistency of our findings in this analysis and previous studies support future investigations that can elucidate the underlying differences in biology, physiology, and pathology between subphenotypes. Promisingly, introduction of technology that permits bedside identification of subphenotypes may lead to an era of subphenotype-based and biology-specific clinical trials, which in turn could significantly impact patient outcomes. 
Reference:

1. Frank AJ, Thompson BT, (2010) Pharmacological treatments for acute respiratory distress syndrome. Curr Opin Crit Care 16: 62-68

2. Bosma KJ, Taneja R, Lewis JF, (2010) Pharmacotherapy for prevention and treatment of acute respiratory distress syndrome: current and experimental approaches. Drugs 70: 1255-1282

3. Jacobson JR, Barnard JW, Grigoryev DN, Ma SF, Tuder RM, Garcia JG, (2005) Simvastatin attenuates vascular leak and inflammation in murine inflammatory lung injury. Am J Physiol Lung Cell Mol Physiol 288: L1026-1032

4. Chopra V, Rogers MA, Buist M, Govindan S, Lindenauer PK, Saint S, Flanders SA, (2012) Is statin use associated with reduced mortality after pneumonia? A systematic review and meta-analysis. Am J Med 125: 1111-1123

5. Shyamsundar M, McKeown ST, O'Kane CM, Craig TR, Brown V, Thickett DR, Matthay MA, Taggart CC, Backman JT, Elborn JS, McAuley DF, (2009) Simvastatin decreases lipopolysaccharide-induced pulmonary inflammation in healthy volunteers. Am J Respir Crit Care Med 179: 1107-1114

6. McAuley DF, Laffey JG, O'Kane CM, Perkins GD, Mullan B, Trinder TJ, Johnston P, Hopkins PA, Johnston AJ, McDowell C, McNally C, Investigators H-, Irish Critical Care Trials G, (2014) Simvastatin in the acute respiratory distress syndrome. N Engl J Med 371: 1695-1703

7. National Heart L, Blood Institute ACTN, Truwit JD, Bernard GR, Steingrub J, Matthay MA, Liu KD, Albertson TE, Brower RG, Shanholtz C, Rock P, Douglas IS, deBoisblanc BP, Hough $\mathrm{CL}$, Hite RD, Thompson BT, (2014) Rosuvastatin for sepsis-associated acute respiratory distress syndrome. N Engl J Med 370: 2191-2200

8. Calfee CS, Delucchi K, Famous KR, (2017) Reply: Next Step to Understanding Subphenotypes of Acute Respiratory Distress Syndrome. Am J Respir Crit Care Med 196: 796

9. Famous KR, Delucchi K, Ware LB, Kangelaris KN, Liu KD, Thompson BT, Calfee CS, Network A, (2017) Acute Respiratory Distress Syndrome Subphenotypes Respond Differently to Randomized Fluid Management Strategy. Am J Respir Crit Care Med 195: 331-338

10. Calfee CS, Delucchi KL, Sinha P, Matthay MA, Hackett J, Shankar-Hari M, McDowell C, Laffey JG, O'Kane CM, McAuley DF, Irish Critical Care Trials G, (2018) Acute respiratory distress syndrome subphenotypes and differential response to simvastatin: secondary analysis of a randomised controlled trial. Lancet Respir Med 6: 691-698

11. Delucchi K, Famous KR, Ware LB, Parsons PE, Thompson BT, Calfee CS, Network A, (2018) Stability of ARDS subphenotypes over time in two randomised controlled trials. Thorax

12. Calfee CS, Delucchi K, Parsons PE, Thompson BT, Ware LB, Matthay MA, Network NA, (2014) Subphenotypes in acute respiratory distress syndrome: latent class analysis of data from two randomised controlled trials. Lancet Respir Med 2: 611-620 
13. Calfee CS, Delucchi KL, Sinha P, Matthay MA, Hackett J, Shankar-Hari M, McDowell C, Laffey JG, O'Kane CM, McAuley DF, (2018) ARDS Subphenotypes and Differential Response to Simvastatin: Secondary Analysis of a Randomized Controlled Trial . Lancet Respir Med: [In Press]

14. Sinha P, Delucchi K, Thompson BT, McAuley DF, Matthay MA, Calfee CS (2018) Latent Class Analysis of Acute Respiratory Distress Syndrome (ARDS): Secondary Analysis of the Statins for Acutely Injured Lungs from Sepsis (SAILS) TrialAmerican Journal of Respiratory and Critical Care Medicine, pp. A6187-A6187

15. Muthén LK, Muthén BO, (2017) Mplus user's guide (version 8). Los Angeles, CA

16. Pierrakos C, Vincent JL, (2012) The changing pattern of acute respiratory distress syndrome over time: a comparison of two periods. Eur Respir J 40: 589-595

17. Wurpts IC, Geiser C, (2014) Is adding more indicators to a latent class analysis beneficial or detrimental? Results of a Monte-Carlo study. Front Psychol 5: 920

18. Maiolo G, Collino F, Vasques F, Rapetti F, Tonetti T, Romitti F, Cressoni M, Chiumello D, Moerer O, Herrmann P, Friede T, Quintel M, Gattinoni L, (2018) Reclassifying Acute Respiratory Distress Syndrome. Am J Respir Crit Care Med 197: 1586-1595

19. Nuckton TJ, Alonso JA, Kallet RH, Daniel BM, Pittet JF, Eisner MD, Matthay MA, (2002) Pulmonary dead-space fraction as a risk factor for death in the acute respiratory distress syndrome. N Engl J Med 346: 1281-1286

20. Warren MA, Zhao Z, Koyama T, Bastarache JA, Shaver CM, Semler MW, Rice TW, Matthay MA, Calfee CS, Ware LB, (2018) Severity scoring of lung oedema on the chest radiograph is associated with clinical outcomes in ARDS. Thorax 73: 840-846

21. Landesberg G, Levin PD, Gilon D, Goodman S, Georgieva M, Weissman C, Jaffe AS, Sprung CL, Barak V, (2015) Myocardial Dysfunction in Severe Sepsis and Septic Shock: No Correlation With Inflammatory Cytokines in Real-life Clinical Setting. Chest 148: 93-102

22. Prescott HC, Calfee CS, Thompson BT, Angus DC, Liu VX, (2016) Toward Smarter Lumping and Smarter Splitting: Rethinking Strategies for Sepsis and Acute Respiratory Distress Syndrome Clinical Trial Design. Am J Respir Crit Care Med 194: 147-155

23. Davenport EE, Burnham KL, Radhakrishnan J, Humburg P, Hutton P, Mills TC, Rautanen A, Gordon AC, Garrard C, Hill AV, Hinds CJ, Knight JC, (2016) Genomic landscape of the individual host response and outcomes in sepsis: a prospective cohort study. Lancet Respir Med 4: 259-271

24. Sweeney TE, Azad TD, Donato M, Haynes WA, Perumal TM, Henao R, Bermejo-Martin JF, Almansa R, Tamayo E, Howrylak JA, Choi A, Parnell GP, Tang B, Nichols M, Woods CW, Ginsburg GS, Kingsmore SF, Omberg L, Mangravite LM, Wong HR, Tsalik EL, Langley RJ, Khatri $P$, (2018) Unsupervised Analysis of Transcriptomics in Bacterial Sepsis Across Multiple Datasets Reveals Three Robust Clusters. Crit Care Med 46: 915-925

25. Scicluna BP, van Vught LA, Zwinderman AH, Wiewel MA, Davenport EE, Burnham KL, Nurnberg P, Schultz MJ, Horn J, Cremer OL, Bonten MJ, Hinds CJ, Wong HR, Knight JC, van der Poll T, consortium M, (2017) Classification of patients with sepsis according to blood genomic endotype: a prospective cohort study. Lancet Respir Med 5: 816-826

26. Wong HR, Cvijanovich N, Lin R, Allen GL, Thomas NJ, Willson DF, Freishtat RJ, Anas N, Meyer K, Checchia PA, Monaco M, Odom K, Shanley TP, (2009) Identification of pediatric septic shock subclasses based on genome-wide expression profiling. BMC Med 7: 34 
27. Siempos, II, Maniatis NA, Kopterides P, Magkou C, Glynos C, Roussos C, Armaganidis A, (2010) Pretreatment with atorvastatin attenuates lung injury caused by high-stretch mechanical ventilation in an isolated rabbit lung model. Crit Care Med 38: 1321-1328

28. Craig TR, Duffy MJ, Shyamsundar M, McDowell C, O'Kane CM, Elborn JS, McAuley DF, (2011) A randomized clinical trial of hydroxymethylglutaryl- coenzyme a reductase inhibition for acute lung injury (The HARP Study). Am J Respir Crit Care Med 183: 620626

29. Sahebkar A, Rathouska J, Simental-Mendia LE, Nachtigal P, (2016) Statin therapy and plasma cortisol concentrations: A systematic review and meta-analysis of randomized placebo-controlled trials. Pharmacol Res 103: 17-25

30. Sahebkar A, Serban C, Ursoniu S, Mikhailidis DP, Undas A, Lip GY, Bittner V, Ray K, Watts GF, Hovingh GK, Rysz J, Kastelein JJ, Banach M, Lipid, Blood Pressure Meta-analysis Collaboration G, (2016) The impact of statin therapy on plasma levels of von Willebrand factor antigen. Systematic review and meta-analysis of randomised placebo-controlled trials. Thromb Haemost 115: 520-532

31. Bonsu KO, Reidpath DD, Kadirvelu A, (2015) Effects of Statin Treatment on Inflammation and Cardiac Function in Heart Failure: An Adjusted Indirect Comparison Meta-Analysis of Randomized Trials. Cardiovasc Ther 33: 338-346

32. Smith MEB, Lee NJ, Haney E, Carson S (2009) Drug Class Review: HMG-CoA Reductase Inhibitors (Statins) and Fixed-dose Combination Products Containing a Statin: Final Report Update 5, Portland (OR)

33. Lee CC, Lee MG, Hsu TC, Porta L, Chang SS, Yo CH, Tsai KC, Lee M, (2018) A PopulationBased Cohort Study on the Drug-Specific Effect of Statins on Sepsis Outcome. Chest 153: 805-815

34. Mansur A, Steinau M, Popov AF, Ghadimi M, Beissbarth T, Bauer M, Hinz J, (2015) Impact of statin therapy on mortality in patients with sepsis-associated acute respiratory distress syndrome (ARDS) depends on ARDS severity: a prospective observational cohort study. BMC Med 13: 128

35. Lobo SM, Lobo FR, Bota DP, Lopes-Ferreira F, Soliman HM, Melot C, Vincent JL, (2003) Creactive protein levels correlate with mortality and organ failure in critically ill patients. Chest 123: 2043-2049

36. Castell JV, Gomez-Lechon MJ, David M, Fabra R, Trullenque R, Heinrich PC, (1990) Acutephase response of human hepatocytes: regulation of acute-phase protein synthesis by interleukin-6. Hepatology 12: 1179-1186

37. Bajwa EK, Khan UA, Januzzi JL, Gong MN, Thompson BT, Christiani DC, (2009) Plasma Creactive protein levels are associated with improved outcome in ARDS. Chest 136: 471480

38. Pierrakos C, Vincent JL, (2010) Sepsis biomarkers: a review. Crit Care 14: R15

39. Navarro SL, Kantor ED, Song X, Milne GL, Lampe JW, Kratz M, White E, (2016) Factors Associated with Multiple Biomarkers of Systemic Inflammation. Cancer Epidemiol Biomarkers Prev 25: 521-531

40. Mackenzie I, Woodhouse J, (2006) C-reactive protein concentrations during bacteraemia: A comparison between patients with and without liver dysfunction. Intensive Care Med 32: 1344-1351 
41. Bos LD, Schouten LR, van Vught LA, Wiewel MA, Ong DSY, Cremer O, Artigas A, MartinLoeches I, Hoogendijk AJ, van der Poll T, Horn J, Juffermans N, Calfee CS, Schultz MJ, consortium M, (2017) Identification and validation of distinct biological phenotypes in patients with acute respiratory distress syndrome by cluster analysis. Thorax 72: 876883 


\section{Figures Caption}

Fig. 1 Standardized values for continuous class-predicting variables. The variables are sorted from left to right in descending order for the difference in values between the hyper-

inflammatory and hypo-inflammatory subphenotype. Standardized values were calculated by assigning the mean of the variables as 0 and standard deviation as $1 . \mathrm{BMI}=$ body mass index; $\mathrm{SBP}=$ systolic blood pressure; ICAM-1 = intercellular adhesion molecule-1; IL-6 = Interleukin 6; $\mathrm{IL}-8$ = Interleukin 8; Mean Air Press = mean airway pressure; PAI-1 = plasminogen activator inhibitor-1; PEEP = positive end-expiratory pressure; Plat Press = plateau pressure; sTNFr1 = tumor necrosis factor receptor-1; $\mathrm{VE}=$ minute ventilation; $\mathrm{VT}=$ tidal volume; $\mathrm{WBC}=$ white blood cell count.

Fig. 2 Kaplan-Meier survival curves to 60 days for patients in the SAILS trial; the population was stratified by ARDS subphenotype and treatment (rosuvastatin vs placebo). Comparison of curves using Cox Proportional Hazards modeling; $p=0.45$ subphenotype 1 (hypoinflammatory), $p=0.331$ subphenotype 2 (hyper-inflammatory). 